\title{
Representación semántica de eventos sobre seguridad: un enfoque basado en lingüística
}

\author{
José A. Reyes-Ortiz \\ Universidad Autónoma Metropolitana, \\ Departamento de Sistemas, México \\ jaro@correo.azc.uam.mx
}

\begin{abstract}
Resumen. Este artículo reporta la elaboración de una ontología mediante un proceso basado en el principio de modularización. El proceso considera la modularización, la cual consiste en dividir un sistema de ontologías en ontologías individuales con la finalidad de facilitar la reutilización, mantenimiento e integración de ontologías. El principal objetivo de este artículo es diseñar un sistema de ontologías para la representación de eventos sobre seguridad apoyado en consideraciones lingüísticas, el cual se enfoca en la creación de módulos desde el inicio del proceso de diseño de ontologías. El sistema de ontologías es creado de manera modular y evaluado utilizando una fase de axiomatización.
\end{abstract}

Palabras clave: Eventos sobre seguridad, creación modular de ontologías, representación de conocimiento, aspectos lingüísticos.

\section{Semantic Representation of Security Events: A Linguistic-based Approach}

\begin{abstract}
This paper reports the development process of an ontology based on the principle of modularization. The purpose of considering a modularization is to divide the whole system of ontologies into individual ontologies in order to facilitate reuse, maintenance and integration of them. Therefore, the main aim of this paper is to design a set of ontologies for representing security-related events, which is supported on linguistic issues and a modular design process is conducted from the beginning of such process. A set of individual ontologies is created in a modular way, they are integrated to form the ontological system, and then, it is evaluated with an instantiation phase.
\end{abstract}

Keywords. Security events, modular design ontologies, knowledge representation, linguistic aspects. 


\section{Introducción}

La cantidad de textos periodísticos en la Web está creciendo todos los días. Este tipo de textos contienen eventos en la mayoría de sus párrafos, los cuales describen sucesos y un conjunto de dimensiones, a saber: lugar del suceso, tiempo en que ocurrió el suceso, causas y objetos o agentes involucrados. Con la proliferación de este tipo de textos, son necesarias, cada día con mayor urgencia, herramientas de extracción automática, representación y búsqueda de información centrada en eventos. La representación de eventos es un factor clave en este conjunto de herramientas ya que provee los mecanismos para ayudar a la extracción de información sobre eventos en textos periodísticos no estructurados y hacer posible su búsqueda.

Un modelo ontológico se debe considerar para lograr una representación de eventos basada en su significado y con una estructura semántica capaz de lograr inferencias y localización automática de información. Las ontologías son capaces de proveer los mecanismos necesarios para afrontar la problemática descrita arriba, ellas son un modelo computacional que se utiliza para la representación de información de un dominio específico, sus propiedades y como se relacionan los conceptos [1].

Este artículo se centra en resolver la carencia de una estructura semántica procesable por computadoras con información de eventos reales. Esta representación considera el principio de modularización, reportado en [2], en el proceso inicial de construcción de un sistema de ontologías para la representación de eventos sobre seguridad y proporcionar un repositorio estructurado semánticamente de eventos. Este resultado puede ser útil para el análisis automatizado de información sobre eventos y lograr vincular datos de eventos dispersos en la Web.

El resto del artículo está organizado de la siguiente manera. En la Sección 2 se presentan los trabajos relacionados con la representación de información utilizando ontologías. La Sección 3 presenta las teorías sobre la cognición de eventos en general y de manera específica se aterriza en eventos sobre seguridad. La creación del sistema de ontologías para la representación de eventos sobre seguridad basada en aspectos lingüísticos, se muestra en la Sección 4. La axiomatización del sistema de ontologías se expone en la Sección 5, con la finalidad de validar y verificar dicho sistema. Finalmente, las conclusiones y el trabajo a futuro son presentados en la Sección 6.

\section{Trabajos relacionados}

La tarea de representación de conocimiento ha presentado avances significativos en los últimos años, esto gracias a la incorporación de ontologías como mecanismo de representación. Por ello, en esta sección se presentan los trabajos relacionados con la representación de eventos utilizando ontologías.

En esta área de investigación, se han presentado diversos trabajos como en [3], donde se expone un método para la extracción de eventos violentos a partir de noticias en línea con el propósito de instanciar una ontología, en el cual se utilizan patrones textuales y agrupamiento de textos. También, es el caso del trabajo presentado en [4] que desarrolla 
una ontología del dominio de seguridad de la información para describir y representar eventos ocasionados por ataques cibernéticos o software maliciosos.

En [5] se reporta el proceso de construcción de una ontología para resolución de identidades en el dominio de la salud pública mediante la representación de eventos de nacimientos, decesos, certificados de registros y reportes mixtos de salud. La idea de los autores es solucionar los problemas de heterogeneidad estructural y semántica al vincular fuentes de datos dispersas, utilizando una ontología que funcione como un repositorio semántico, el cual proporcione una visión de cómo la identidad de un individuo evoluciona con el tiempo.

Por su parte, en [6] se describe un sistema que reconoce eventos a partir de noticias. El sistema presentado clasifica noticias y genera instancias de clases definidas en una ontología creada manualmente. Los autores crean una topología de eventos, con relaciones semánticas entre eventos y propiedades, para posteriormente, desempeñar un proceso de extracción de conocimiento con la finalidad de poblar la ontología.

El trabajo presentado en [7] tiene por objetivo el descubrimiento y representación semiautomático de eventos financieros utilizando patrones léxicos-semánticos a partir de textos. Sin embargo, los autores no crean una ontología con los datos de eventos descubiertos, por su parte, ellos crean una base de conocimiento con la información de eventos extraída de textos sobre finanzas.

En el dominio de la medicina, específicamente, en el dominio de fármacos, diversos trabajos han propuesto la representación, extracción y recuperación de eventos adversos provocados por los fármacos utilizando ontologías para apoyar el proceso de toma de decisiones de los médicos [8, 11].

Este trabajo centra su aportación en la incorporación de la modularización desde el inicio del proceso de construcción de un sistema de ontologías para la representación de eventos sobre seguridad en español. La idea es que este sistema de ontologías apoye la tarea de extracción automática de eventos a partir de noticias en español.

\section{Cognición de eventos sobre seguridad}

La cognición se refiere a la forma en que la mente (cerebro) conoce y percibe el mundo. Por su parte, los procesos cognitivos hacen referencia a la adquisición de conocimiento a partir de la percepción otorgando un significado y una organización a la información percibida.

El proceso cognitivo ha sido estudiado en diferentes campos incluyendo la neurología, psicología, sociología y filosofía. En la actualidad, estos estudios se han trasladado a las ciencias computacionales y ha despertado un interés particular en la inteligencia artificial, la representación de conocimiento y el aprendizaje automático.

Los eventos intervienen en el proceso cognitivo desde que estos ayudan a organizar la información percibida y otorgan un orden espacio-temporal, además sitúan a los protagonistas que aparecen en dicha información. De esta manera, los eventos han sido concebidos desde el proceso cognitivo, como un suceso que involucra un cambio de estado, donde se involucran aspectos locativos, temporales y causales [12]. 
Este artículo se centra en la cognición de eventos sobre seguridad, donde los eventos son el núcleo sobre el cual actúan las dimensiones (espacialidad, temporalidad, protagonistas y causalidad), esto se debe a su intervención e importancia en la forma que los humanos perciben y estructuran la información durante el proceso de comprensión del conocimiento (proceso cognitivo).

De esta cognición se obtiene una definición formal del concepto Evento y sus elementos, la cual se muestra en la ecuación 1:

$$
\text { Evento }=(E, S, T, C, P),
$$

compuesta por una etiqueta $E$ del evento; $S, T, C$ y $P$ corresponde a las dimensiones de espacialidad, temporalidad, causalidad y protagonistas. La información de las dimensiones constituye los complementos del evento, los cuales agregan significado y organización a los eventos.

Los eventos son los puntos focales de las situaciones del mundo (textos, imágenes, audio, hechos, entre otras), por lo tanto, al intentar comprenden estas situaciones, el receptor construye una representación de los protagonistas, eventos, estados, acciones, relaciones, espacio, tiempo, causas y efectos.

Los textos en lenguaje natural son una forma de comunicar las situaciones, por lo tanto, se comprensión involucra poner una atención focal en los eventos descritos con la finalidad de crear una representación de lo que se expresa en términos espacio, tiempo, causas y protagonistas. En estos textos, la estructura lingüística actúa como un medio para expresar cómo está construida la situación o el mundo.

Los eventos sobre seguridad, como acaecimientos, están presentes en la mayoría de los textos periodísticos. En estos textos, los eventos están representados por una frase verbal (L. Tesnière [13], M. Halliday [14]). Tanto la teoría de L. Tesnière como la de M. Halliday afirman que el verbo es el núcleo sobre el cual giran todos los elementos de la oración, los cuales se dividen en actantes (agente, objeto, y beneficiario) y los circunstantes (instrumento, fuerza, tiempo y locativo).

Los actantes, en los eventos sobre seguridad, responden a preguntas como: ¿quién realizó el hallazgo?, ¿con qué realizó el ataque?, ¿quién fue arrestado?, por mencionar algunas.

La presencia de estos actantes en los eventos sobre seguridad depende en gran medida del tipo de evento y el verbo que lo caracteriza, y por consecuencia, el número de valencias necesarias. Estas valencias cambian de acuerdo al contexto y al significado del verbo. Para percibir esta información, se presenta la teoría de valencias de Tesnière ([13]), haciendo un énfasis en los valores actanciales de los verbos.

La teoría de Tesnière afirma que la valencia de los verbos es el número de actantes que puede recibir para producir un significado coherente. Esta teoría se aplica a diversas lenguas neolatinas como el español.

El análisis de valencias de los verbos permite comprender oraciones ambiguas. Por lo tanto, todo verbo tiene su propio valor actancial, los cuales se clasifican, según [13], en los siguientes grupos.

a. Verbos avalantes. Aquellos verbos que no tienen actantes y su significado no se ve afectado por esta circunstancia, ejemplos de estos verbos son: llover, 
nevar, tronar (verbos de tiempo atmosférico), ser (en expresiones como ser tarde, ser necesario, ser lícito), haber (con valor impersonal).

b. Verbos monovalentes. Verbos que requieren un actante, comúnmente el sujeto sintáctico que puede ser alguien o algo. Como ejemplos de estos verbos se tiene salir, dormir, suicidar.

c. Verbos bivalentes. Los verbos que requieren necesariamente la presencia de dos actantes, la ausencia del segundo actante representa una mutilación del significado del verbo.

d. Verbos trivalentes. Verbos que requieren la presencia de tres actantes, como el caso de los verbos poner y dar.

En la Figura 1 se muestra un ejemplo para cada una de las cuatro categorías de verbos descritas anteriormente. De esta manera, la Figura 1(a) muestra el ejemplo del verbo llover con el esquema actancial avalante (sin actores), la Figura 1(b) expone el ejemplo para el verbo dormir en el esquema actancial monovalente (con un actor), la Figura 1(c) muestra el ejemplo de un esquema actancial bivalente (con dos actores) mediante el verbo comer, finalmente, la Figura 1(d) expone un ejemplo del esquema actancial trivalente (con tres actores) usando el verbo dar.

llover

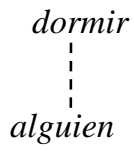

(a)<smiles>CC(C)C</smiles>

(c)<smiles>CC(C)(C)[Ge]</smiles>

(d)

Fig. 1. Ejemplo del uso de verbos en cada categoría: avalante, monovalente, bivalente y trivalente.

La idea de los eventos sobre seguridad como un núcleo forma parte de las bases de este artículo, ya que ayudan a construir un modelo de representación durante la comprensión de los textos y la adquisición de conocimiento. Por ello, estas teorías de eventos otorgan el conocimiento fundamental para la creación del sistema de ontologías de eventos relacionados con la seguridad. Las ontologías individuales presentadas en la Sección 4 se basan en esta cognición de eventos.

\section{Creación del sistema de ontologías con bases lingüísticas}

En esta sección se presenta el diseño y la creación del sistema de ontologías para la representación de eventos. En este proceso se considera el aspecto de la modularización, el cual consiste, primero, en crear ontologías individuales y, después, integrarlas con la finalidad de construir el sistema de ontologías. Por su parte, la creación de ontologías individuales considera el aspecto de la reutilización, es decir, buscar ontologías disponibles que resuelvan el problema particular.

En nuestro caso, el sistema de ontologías para la representación de eventos sobre seguridad se compone de tres módulos (ontologías individuales): ontología de tiempo, 
ontología de espacio y ontología de eventos sobre seguridad, la cual se comporta como el núcleo del sistema de representación.

La ontología de tiempo y la ontología de espacio son ontologías reutilizadas y adaptadas de la literatura, mientras que, la ontología núcleo sobre eventos de seguridad es diseñada y creada desde cero.

En el proceso de creación de la ontología núcleo se consideran aspectos lingüísticos y las características sintácticas de los eventos sobre seguridad con el apoyo de la presencia de este tipo de eventos en textos de noticias.

Se utiliza la sintaxis de Manchester para OWL 1.1 [15] con el propósito de presentar las ontologías individuales y, posteriormente, el sistema de ontologías para la representación semántica de conocimiento en una sintaxis amigable para el usuario. La creación de las tres ontologías individuales se describe a continuación.

\subsection{Ontología de tiempo}

Los eventos tienen, entre sus características o complementos, al tiempo, que responde a la pregunta ¿cuándo sucedió el evento? Para considerar esta característica en los eventos se ha propuesto la reutilización y adaptación de la ontología llamada TimeOntology [16]. Esta ontología individual es adaptada al español para su integración, más adelante, en el sistema de ontologías.

La ontología adaptada tiene las siguientes clases.

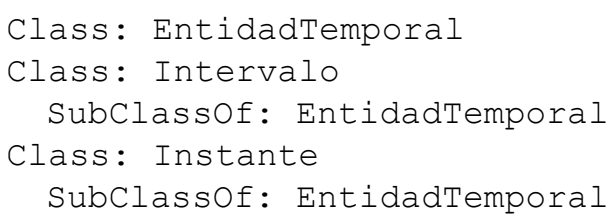

La clase Intervalo se utiliza para representar una entidad temporal con una extensión o duración. Por su parte, la clase Instante es utilizada para representar una entidad temporal con una porción breve de tiempo.

Esta ontología individual será integrada con la ontología de eventos sobre seguridad a través de una relación que será descrita más adelante.

\subsection{Ontología de espacio}

El espacio es una característica primordial de los eventos, desde que éste expresa el lugar donde se realiza el suceso, respondiendo a la pregunta ¿dónde sucedió? En este rubro se ha adaptado una ontología espacial como módulo (ontología individual) para el sistema de ontologías propuestas en este artículo. Así pues, se ha adecuado la ontología llamada OntoEspacio [17], la cual considera la clase Espacio como núcleo y a partir de ella, surgen subclases o tipos de espacios.

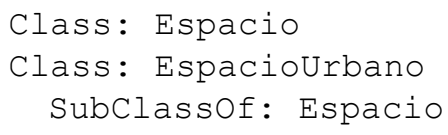




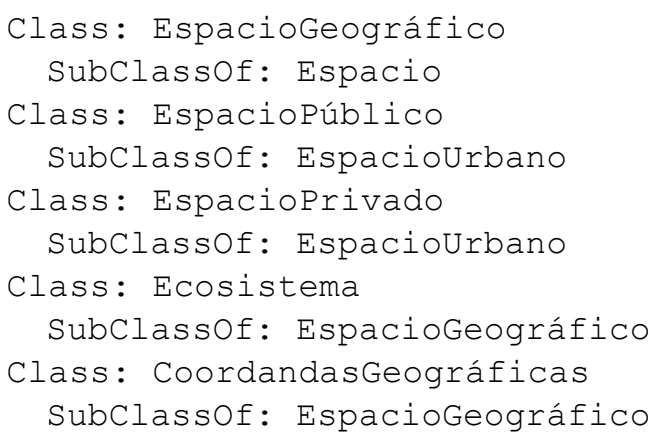

Esta ontología individual será integrada con la ontología de eventos sobre seguridad a través de una relación que será descrita más adelante.

\subsection{Ontología núcleo de eventos sobre seguridad}

La ontología núcleo considera a los eventos sobre seguridad como su parte primordial. Es por ello que esta ontología se basa en la clase llamada EventoSeguridad de la cual se desprenden cuatro subclases, es decir, en este trabajo se consideran cuatro tipos de eventos sobre seguridad.

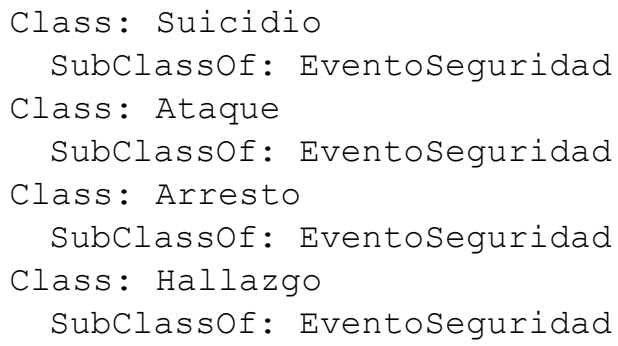

Adicionalmente se define un conjunto de clases para representar los actantes de la teoría de Tesnière [13]. A partir de dichas teorías se obtienen las clases Actor y Objeto, de las cuales se desprenden las siguientes subclases.

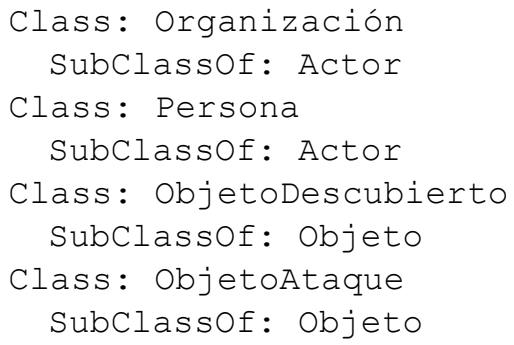

Los cuatro tipos de eventos sobre seguridad y los actantes tienen una serie de relaciones semánticas o relaciones ontológicas. Estas relaciones están basadas en la teoría de eventos y las valencias de los verbos presentadas anteriormente. 
Con estas bases lingüísticas se caracteriza al evento Suicidio como un evento o verbo monovalante, lo cual genera una sola relación entre el evento y un actor.

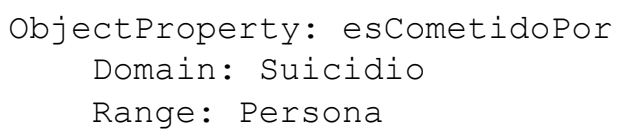

En la Figura 2(a) se puede observar el esquema actancial del evento Suicidio y en la Figura 2(b) se presenta un ejemplo de un evento de este tipo.<smiles>CC(C)(Cl)I</smiles>

(a)

\section{$X$ se suicida}

(b)

Fig. 2. Esquema actancial monovalente del evento Suicidio.

Por su parte, el evento Ataque se caracteriza como un evento o verbo trivalente en el cual intervienen el atacante (persona que realiza el ataque), el atacado (persona lastimada en el ataque) y el instrumento (objeto o herramienta con la cual fue realizado el ataque). Por lo tanto, tres relaciones son generadas a partir de este tipo de evento.

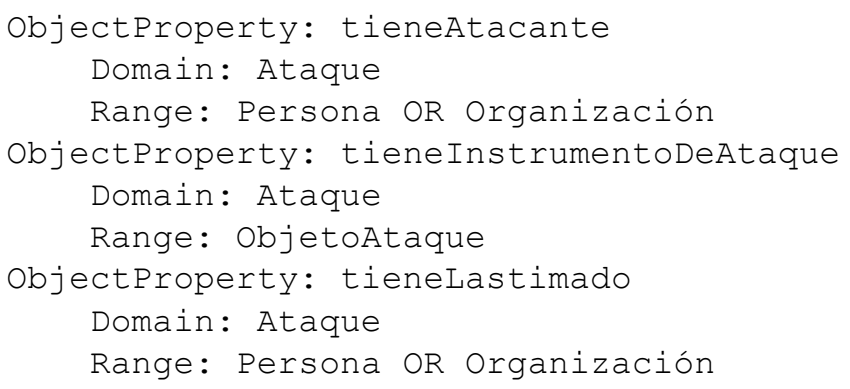

En la Figura 3(a) se puede observar el esquema actancial del evento Ataque y en la Figura 3(b) se presenta un ejemplo de este tipo de evento.

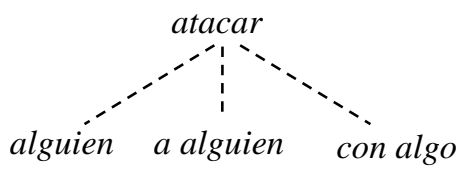

(a)

\section{$X$ ataca a $Y$ utilizando $Z$}

(b)

Fig. 3. Esquema actancial trivalente del evento Ataque.

Con respecto al evento de tipo Hallazgo o Descubrimiento se han caracterizado dos relaciones entre el evento y los actantes. De esta manera, se tiene que este tipo de evento 
es considerado con un valor actancial bivalente, lo cual origina las siguientes relaciones en el modelo ontológico.

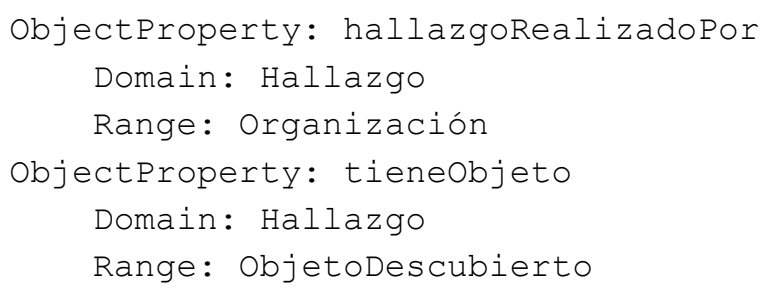

En la Figura 4(a) se puede observar el esquema actancial del evento Hallazgo con una relación hacia la clase Organización (quien realiza el hallazgo o descubrimiento) y una segunda relación hacia un Objeto (elemento encontrado). Por su parte, en la Figura 4(b) se presenta un ejemplo de esta categoría de eventos.

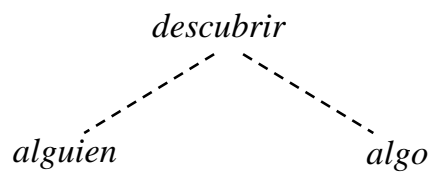

(a)

\section{$X$ descubre $Y$}

(b)

Fig. 4. Esquema actancial bivalente del evento Descubrimiento.

El evento de tipo Arresto se ha caracterizado con un esquema actancial bivalente. Por ello, se representan dos relaciones semánticas, una con la clase Organización (quien realiza el arresto) y otra relación para indicar al arrestado (persona que es detenida). Esto genera el siguiente código en la ontología.

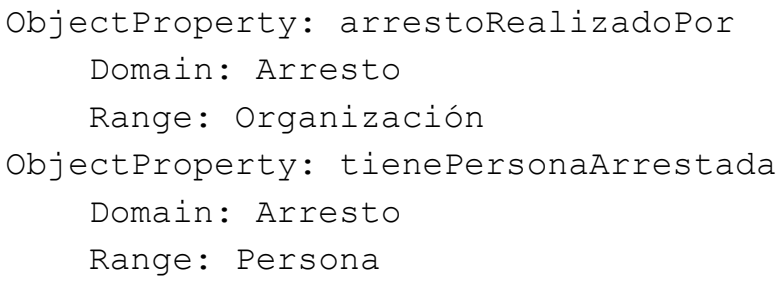

En la Figura 5(a) se puede observar el esquema actancial del evento Arresto y en la Figura 5(b) se presenta un ejemplo de esta clase de eventos.

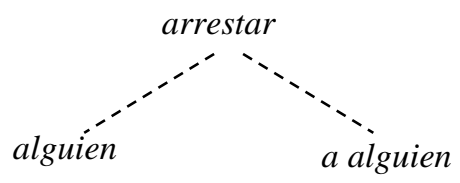

(a)

\section{$X$ arresta a $Y$}

(b)

Fig. 5. Esquema actancial bivalente del evento Arresto. 


\subsection{Integración de ontologías individuales}

El proceso de integración corresponde a la creación de relaciones semánticas entre las ontologías individuales, ya sea que fueron creadas desde cero o adaptadas de ontologías existentes. El objetivo de este proceso es proveer una solución integral, con un sistema de ontologías, a la representación de eventos sobre seguridad considerando sus dimensiones de tiempo, espacio y actores.

Esta integración da origen a la relación llamada sucedeEn, la cual se utiliza para representar el lugar donde sucede el evento sobre seguridad. Esta relación se crea en el sistema de ontologías de la siguiente manera.

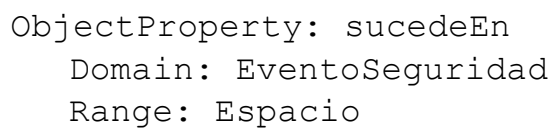

Adicionalmente se crea la relación tieneTiempo, la cual se utiliza para responder a la pregunta ¿cuándo sucedió el evento sobre seguridad? Esta relación se crea en el sistema de ontologías.

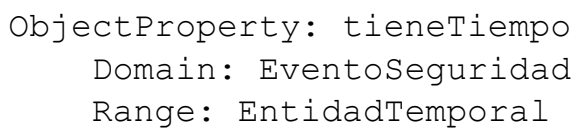

De esta manera quedan integradas las tres ontologías individuales (ontología de espacio, ontología de tiempo y ontología de eventos) con la finalidad de crear un sistema de ontologías con relaciones semánticas entre ellas. El esquema general del sistema de ontologías se puede observar en la Figura 6.

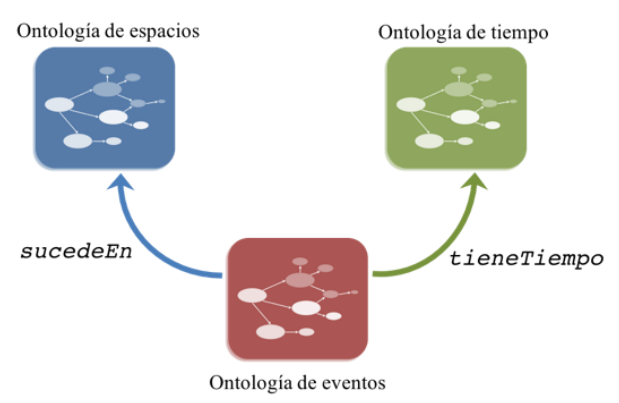

Fig. 6. Integración entre las ontologías individuales.

\section{Axiomatización del sistema de ontologías}

El sistema de ontologías creado con sus características para cada clase es instanciado con miembros o |concepto con la finalidad de validar la consistencia de la ontología. Diferentes tipos de axiomas son considerados en el sistema de ontologías para la representación de eventos sobre seguridad, los cuales se describen a continuación. 


\subsection{Axiomas de definición de clases}

Los axiomas de definición de clases se utilizan para restringir los miembros pertenecientes a una clase, es decir, determinar si dos clases tienen elementos disjuntos o no. En nuestro sistema de ontologías, las siguientes restricciones de definición de clases son creadas.

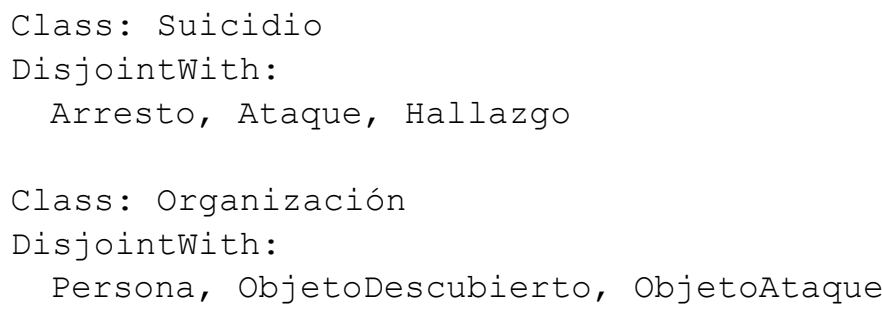

\subsection{Axiomas de definición de propiedades de datos y objetos}

Este tipo de axiomas define restricciones de tipo cardinalidad, existencial, universal y de valor para las características de las clases. Por otro lado, también, define restricciones para establecer que ciertos individuos de una clase tienen relación con individuos de otra clase específica. En este aspecto, se definen una serie de axiomas de restricción para nuestro sistema de ontologías.

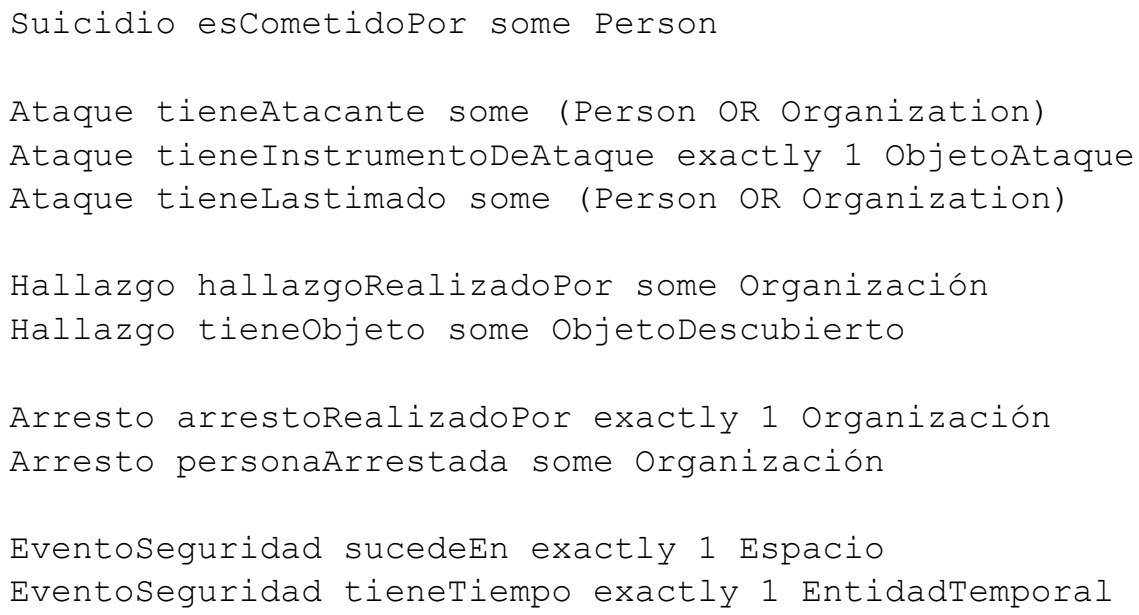

\subsection{Axiomas de poblado de individuos}

El poblado del sistema de ontologías consiste en crear un conjunto de individuos para las clases con la finalidad de hacer posible la evaluación de los axiomas descritos anteriormente. Además, este tipo de axiomas permiten la verificación de la consistencia del sistema de ontologías ya que permiten validar las restricciones de clases, propiedades y objetos. En este aspecto, los siguientes hechos, como axiomas representativos, 
son agregados al sistema ontológico, para los cuales, no se agregan nombres de personas en su lugar se incluyen nombres de instancias anónimas.

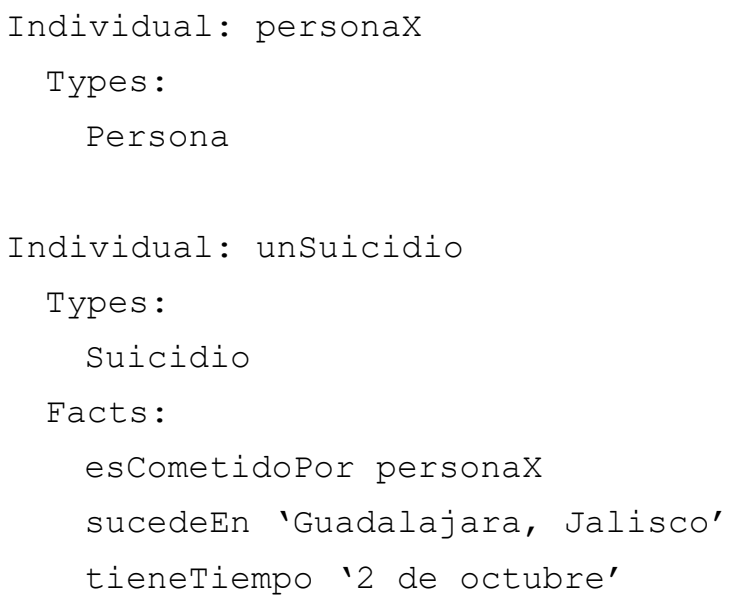

\section{Conclusiones y trabajo futuro}

En este artículo se ha presentado la construcción de un sistema de ontologías para la representación de eventos relacionados con la seguridad. La construcción del sistema de ontologías se ha llevado a cabo siguiendo el principio de modularidad, el cual ha consistido en reutilizar, adaptar y crear tres ontologías individuales. Por un lado, se reutilizan y adaptan de la literatura una ontología de espacio y una ontología de tiempo. Por su parte, la ontología individual sobre eventos de seguridad es creada desde cero considerando la teoría de eventos de [13] con sus características.

Las ontologías individuales creadas son integradas para formar el sistema ontológico general, de esta manera, se obtiene un modelo ontológico para la representación de eventos sobre seguridad, donde se puede representar, adicionalmente, su espacio (lugar donde sucedió) y el tiempo.

La aportación principal de este artículo se centra en lograr la creación de un sistema ontológico, basado en la modularidad, para la representación de eventos relacionados con seguridad. Este sistema puede ser de gran utilidad para apoyar diversas aplicaciones como sistemas de pregunta-respuesta o buscadores semánticos de información sobre eventos.

Como trabajo futuro, se plantea el poblado semiautomático del modelo ontológico de eventos sobre seguridad a partir de noticias de periódicos en español usando patrones lingüísticos, los cuales se podrían obtener de las relaciones semánticas en la ontología. Así como también técnicas de reconocimiento de entidades nombradas para identificar y clasificar las entidades de personas, objetos y organizaciones. Enriquecer las relaciones con sinónimos representa una tarea a futuro que puede ayudar a mejorar la tarea de descubrimiento de eventos a partir de textos. 


\section{Referencias}

1. Weigand, H.: Multilingual ontology-based lexicon for news filtering. In: K. Mahesh, editor, The TREVI Project, 138-159 (1997)

2. Bravo, M., Reyes-Ortiz, J. A., Alcántara, R., Rodríguez, J.: Addresing Clarity and Coherence during Ontology Construcction. In: Proceedings of the Mexican International Conference on Computer Science (2016)

3. Piskorski, J., Tanev, H., Wennerberg, P. O.: Extracting violent events from on-line news for ontology population. In: International Conference on Business Information Systems, 287300 (2007)

4. Obrst, L., Chase, P., Markeloff, R.: Developing an Ontology of the Cyber Security Domain. In: The 11th International Conference on Semantic Technology for Intelligence, Defense, and Security, 49-56 (2012)

5. Duncan, J., Eilbeck, K., Narus, S. P., Clyde, S., Thornton, S., Staes, C.: Building an Ontology for Identity Resolution in Healthcare and Public Health. Online journal of public health informatics 7(2) (2015)

6. Vargas-Vera, M., Celjuska, D.: Event recognition on news stories and semi-automatic population of an ontology. In: Proceedings of the 2004 IEEE/WIC/ACM International Conference on Web Intelligence, 615-618 (2004)

7. Borsje, J., Hogenboom, F., Frasincar, F.: Semi-automatic financial events discovery based on lexico-semantic patterns. International Journal of Web Engineering and Technology, 6(2), 115-140 (2010)

8. Adam, T. J., Wang, J.: Adverse Drug Event Ontology: Gap Analysis for Clinical Surveillance Application. In: AMIA Summits on Translational Science Proceedings, 16-20 (2015)

9. Gurulingappa, H., Mateen-Rajpu, A., Toldo, L.: Extraction of potential adverse drug events from medical case reports. Journal of biomedical semantics, 3, 15 (2012)

10. He, Y., Sarntivijai, S., Lin, Y., Xiang, Z., Guo, A., Zhang, S., Smith, B.: OAE: the ontology of adverse events. Journal of biomedical semantics, 5, 29 (2014)

11. Winnenburg, R., Shah, N. H.: Generalized enrichment analysis improves the detection of adverse drug events from the biomedical literature. BMC bioinformatics, 17, 250 (2016)

12. Miller, G., Johnson-Laird, P.: Language and Perception. Belknap Press, Cambridge, UK (1976)

13. L. Tesnière. Eléments de syntaxe structural. second edition, Librairie Klincksieck, Paris (1976)

14. Halliday, M.: An introduction to functional grammar. 2nd edition, Edward Arnold, London (1994)

15. Horridge, M., Patel-Schneider, P. F.: Manchester syntax for OWL 1.1, OWL: Experiences and Directions. Washington (2008)

16. Hobbs, J. R., Pan, F.: Time ontology in OWL. W3C working draft, 27, 133 (2006) https://www.w3.org/TR/owl-time/

17. Gómez, J. D.: Poblado de ontologías espaciales a partir de texto no estructurado. Tesis de Maestría, Centro Nacional de Investigación y Desarrollo Tecnológico, Morelos, México (2012) 